\title{
Gravity Modeling for Variable Fidelity Environments
}

\author{
Michael M. Madden ${ }^{*}$ \\ NASA, Hampton, VA, 23681
}

\begin{abstract}
Aerospace simulations can model worlds, such as the Earth, with differing levels of fidelity. The simulation may represent the world as a plane, a sphere, an ellipsoid, or a highorder closed surface. The world may or may not rotate. The user may select lower fidelity models based on computational limits, a need for simplified analysis, or comparison to other data. However, the user will also wish to retain a close semblance of behavior to the real world. The effects of gravity on objects are an important component of modeling real-world behavior. Engineers generally equate the term gravity with the observed free-fall acceleration. However, free-fall acceleration is not equal to all observers. To observers on the surface of a rotating world, free-fall acceleration is the sum of gravitational attraction and the centrifugal acceleration due to the world's rotation. On the other hand, free-fall acceleration equals gravitational attraction to an observer in inertial space. Surface-observed simulations (e.g. aircraft), which use non-rotating world models, may choose to model observed free fall acceleration as the "gravity" term; such a model actually combines gravitational attraction with centrifugal acceleration due to the Earth's rotation. However, this modeling choice invites confusion as one evolves the simulation to higher fidelity world models or adds inertial observers. Care must be taken to model gravity in concert with the world model to avoid denigrating the fidelity of modeling observed free fall. The paper will go into greater depth on gravity modeling and the physical disparities and synergies that arise when coupling specific gravity models with world models.
\end{abstract}

\section{Nomenclature and Acronyms}

$=$ acceleration due to gravitation acting on an object

$\vec{\gamma} \quad=$ the gravitational acceleration vector

$\gamma_{\mathrm{r}} \quad=$ radial component of gravitation

$\gamma_{\phi g}=$ latitudinal (geocentric) component of gravitation

$\gamma_{\mathrm{n}}^{\mathrm{s}} \quad=$ the normal component of gravitation on the surface

$\gamma_{\mathrm{t}}^{\mathrm{s}} \quad=$ the tangential component of gravitation on the surface

$\omega=$ rotation of the world about its $\mathrm{z}$ axis

$\phi \quad=$ geodetic latitude

$\phi_{\mathrm{g}} \quad=$ geocentric latitude

$\psi=$ true heading of an object

a $\quad=$ semi-major axis of the world's reference ellipse

$\mathrm{b}=$ semi-minor axis of the world's reference ellipse

$\mathrm{cm} \quad=$ centimeters

$\mathrm{C}_{\mathrm{n}, \mathrm{m}}=$ spherical harmonic coefficient (degree $\mathrm{n}$, order $\mathrm{m}$ ) of the gravitational potential

$\mathrm{C}_{\mathrm{n}} \quad=$ zonal spherical harmonic of the gravitational potential (equal to $\mathrm{C}_{\mathrm{n}, 0}$ )

$\mathrm{e}=$ eccentricity of the world's reference ellipse

$\mathrm{f}=$ flattening of the world surface

$\mathrm{f}_{\mathrm{y}} \quad=$ side force friction in body coordinates

$\mathrm{ft} \quad=$ feet

$\mathrm{k}=$ theoretical normal gravity formula constant

$\mathrm{G}=$ universal gravitation constant

$\bar{g}^{s} \quad=$ mean surface gravity

${ }^{*}$ Aerospace Engineer, Flight Simulation and Software Branch, Mail Stop 125B, Senior Member AIAA. 


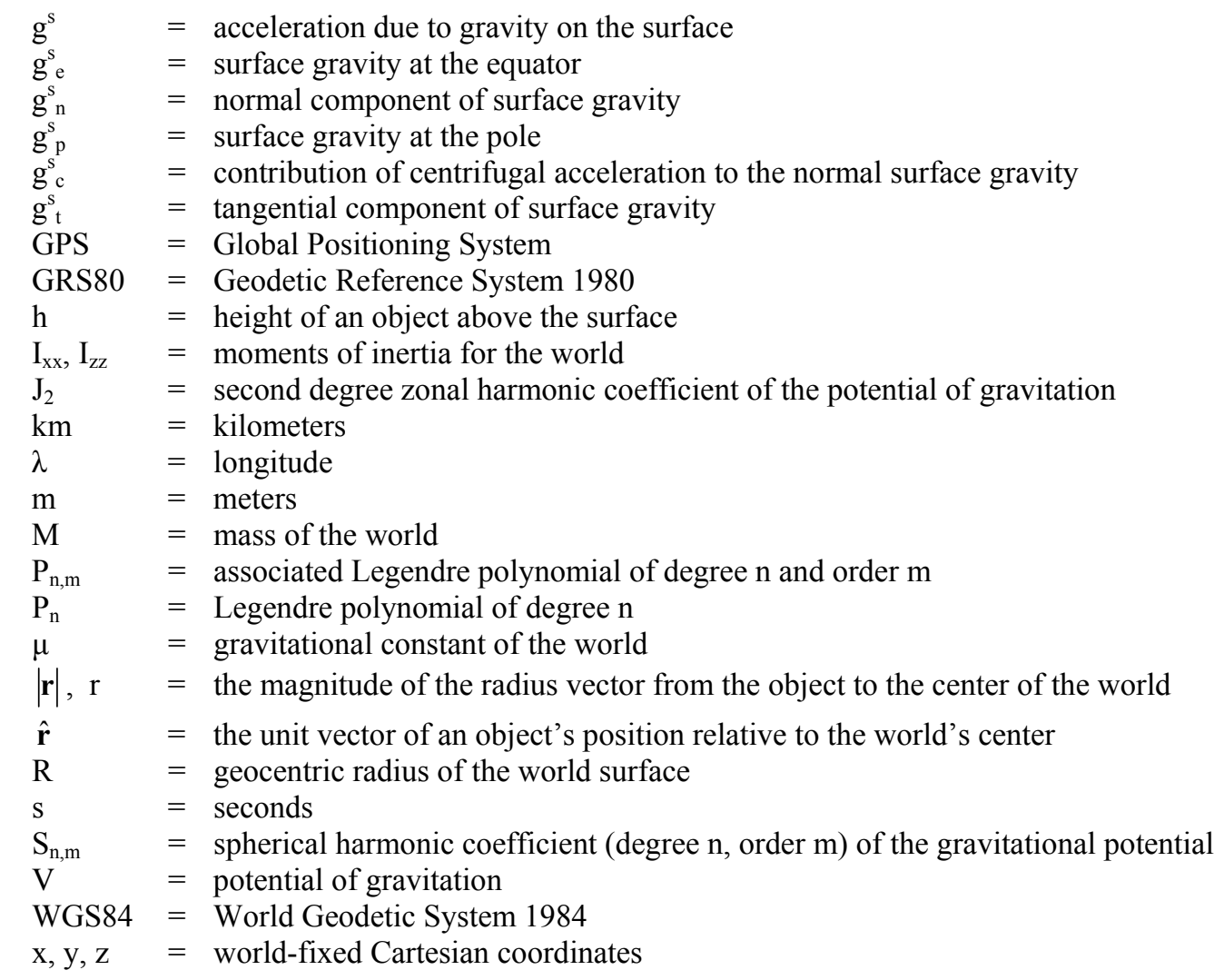

\section{Introduction}

$\mathbf{E}$ or the purposes of this paper, world modeling and gravity modeling are divided into separate entities. The T world model calculates the surface and motion of the world. The modeling of gravity reproduces the observed free-fall acceleration of objects. World and gravity modeling can be accomplished to varying levels of fidelity. The user may select lower fidelity models based on computational limits, a need for simplified analysis, or comparison to other data. However, the user will also wish to retain a close semblance of behavior to the real world. Lower fidelity models may be employed during concept development and the simulation may pass through increasing levels of fidelity as the aerospace products mature. It appears harmless to set the fidelity of the world model and the gravity modeling independently because gravity modeling elements depend on world characteristics that are held constant for a given world model. However, the interaction of gravity modeling elements with the modeled surface can cause combined effects which lower modeling fidelity of near-surface gravity below the fidelity of the individual pieces. The interplay impacts surface interacting simulations such as those that perform ascent, landing, atmospheric flight, and surface travel. This paper presents important concepts for investigating the combination of world and gravity models without reference to a particular world; however, the paper uses the Earth to provide quantitative analysis since its gravity is well understood. The paper begins with a brief overview of world modeling and modeling of gravity. It then performs a detailed examination of combining world and gravity elements of various fidelities. The section on gravity modeling is especially important to understanding the analysis and conclusions. It sets clear distinctions of gravity concepts where colloquial use of the concepts often introduces ambiguities and misconceptions.

\section{World Modeling}

World models simulate the motion of the world and provide an idealized approximation of its shape. Since this paper investigates choices for combining the world model with a gravity model, only the world's motion relative to its center of mass, i.e. its rotation, is relevant. The paper assumes that, like the Earth, the world has a dominant rotation about one axis that will be the $\mathrm{z}$ axis in a world-fixed, Cartesian coordinate system. The paper will focus on the flat-world, sphere, and ellipsoid shape models that are commonly used in modeling the Earth. Higher order shapes like tri-axial ellipsoids can provide improved modeling for a world but are not yet in common use. As one progresses from flat-world to ellipsoid, the idealized shape better approximates the level surface of the world. For this investigation, the ellipsoid model will represent the truth state and is referred to as the reference ellipsoid. 
The flat-world model treats the world as an infinite plane. The flat-world model is valid for short distances that approximate a flat surface on the world. Thus, the flat world is used only in conjunction with models that travel over such short distances (e.g. cars, boats, airplanes). As an infinite plane, the world has no center of mass, but the infinite plane is treated as an equipotential surface of gravity, i.e. possessing a constant potential of gravity. The potential of gravity equals the work to move an object from a given point to infinity in the presence of gravity. The gradient of the potential is the acceleration due to gravity, and the gradient is normal to an equipotential surface.

The sphere model represents the world as a sphere of constant radius. Possible choices for the radius include: the equatorial radius, the average of the equatorial and polar radii. the mean radius, the radius of a sphere of equal surface area to the reference ellipsoid, and the radius of a sphere of equal volume to the reference ellipsoid. The center of mass for the world and the center of the sphere coincide. Except where high navigation accuracy is required, the sphere is a good model for many simulation applications.

The ellipsoid model represents the world as an ellipse of revolution about its semi-minor axis. The ellipsoid is defined as an equipotential surface of the potential of gravity. ${ }^{1}$ Four parameters define its geometry. The two commonly used ellipsoid models are the Geodetic Reference System 1980 (GRS80) and the World Geodetic System 1984 (WGS84). ${ }^{1,2}$ GRS80's parameters are the semi-major axis (a), the Earth's gravitational constant (GM), the second degree zonal gravitational coefficient $\left(\mathrm{J}_{2}\right)^{\dagger}$, and the angular velocity $(\omega)$ of the Earth. WGS84 initially used the equivalent parameters but replaced $J_{2}$ in later revisions with flattening (f) in order to keep flattening constant. Changing flattening would have incurred the "expense of numerous software modifications to GPS receivers and mapping"1 for a minute increase in accuracy. The ellipsoid center and the world's center of mass coincide.

An infinite plane does not rotate; the Earth-fixed frame is an inertial frame. Both the sphere and ellipsoid can model the rotation of the world and rotation will increase the overall fidelity of those models. But, one can also set the rotation to zero for simplicity. Disabling rotation removes the centrifugal acceleration and Coriolis acceleration terms. The centrifugal acceleration is a function of position. Its value near the surface is approximately fixed for a given geodetic latitude. That value is minute; the mean is $0.23 \%$ of gravity. But, the Coriolis acceleration is a function of the object's velocity and remains minuscule only for low speed vehicles (e.g. subsonic). Thus, non-rotating worlds are appropriate for simulations of low-speed vehicles whose travel typically is limited to the world's surface. Section IV explains that turning off rotation has implications for the gravity model of surface-observed simulations.

\section{Gravity Modeling}

Physical geodesy is the science that studies "the gravity field and figure of the earth." 3 Physical geodesy supplies much of the information that simulation engineers use to model the Earth and its gravity. Physical geodesy uses distinguishing definitions for concepts such as gravity and gravitation. Everyday conversation and general physics blurs these distinctions by treating the concepts as interchangeable. The result is ambiguity and misconception. Precise definitions of gravity concepts are necessary to understand the interaction of world modeling and the modeling of gravity. This first half of this section explores the gravity concepts that are important to understanding the models that are defined in the second half.

\section{A. The Relationship between Standard Gravity, Gravity, and Gravitation}

1. What is $9.80665 \mathrm{~m} / \mathrm{s}^{2}$ (a.k.a. $32.174 \mathrm{ft} / \mathrm{s}^{2}$ )?

In 1901, the $3^{\text {rd }}$ General Conference on Weights and Measures (Conférence Générale des Poids et Mesures, CGPM) defined the standard acceleration of gravity to be $980.665 \mathrm{~cm} / \mathrm{s}^{2}$. The chosen value was "already stated in the laws of some countries." ${ }^{4}$ The CGPM established standard gravity as a unit of acceleration that would "put an end to the ambiguity which in current practice still exists on the meaning of the word weight, used sometimes for mass, sometimes for mechanical force." Standard gravity defines a standard weight for a given mass and was the basis for the obsolete unit of kilogram-force ${ }^{\ddagger}$. The aerospace industry continues to use standard gravity as a unit of acceleration called ' $\mathrm{g}$ '. Simulation applications should use standard gravity and not locally modeled gravity when computing accelerations or loads in units of ' $\mathrm{g}$ '. However, standard gravity was defined as a unit, not as an approximation of the Earth's gravity for use in modeling. The value of standard gravity likely derives from the geography of the member states that participated in the 1901 CGPM. The value matches the theoretical gravity on the WGS84 ellipsoid at geodetic latitude $45.5^{\circ}$. But, it is not a value that a modeler might normally choose such as the mean value over the ellipsoid, $9.7976432222 \mathrm{~m} / \mathrm{s}^{2} .{ }^{1}$ Nevertheless, standard gravity has slipped into use as a gravity

\footnotetext{
${ }^{\dagger}$ See section III.B.4 for a definition of $\mathrm{J}_{2}$.

Kilogram-force largely became obsolete when the $11^{\text {th }}$ CGPM (1960) adopted the Newton as the derived unit of force in the SI system.
} 
modeling value. Not all users may be aware that it is an arbitrarily defined unit that derives from gravity; it is not defined as a property of the Earth. Unfortunately, some science and engineering texts inattentively list standard gravity under "properties of the Earth" or "physical constants",5,6 and lend it this mystique.

\section{Gravity and Gravitation}

NASA's educational sites teach that "gravity is a force of attraction that exists between any two masses." A similar definition can also be found in dictionaries and encyclopedias. ${ }^{8-10}$ However, most of our quantitative knowledge of Earth gravity comes from geodesy. In geodesy, the term gravity refers to the free-fall acceleration measured in an Earth-fixed frame. ${ }^{3}$ NASA's Dictionary of Technical Terms for Aerospace Use defines gravity in accordance with geodesy, "Viewed from a frame of reference fixed in the earth, force imparted by the earth to a mass which is at rest relative to the earth. Since the earth is rotating, the force observed as gravity is the resultant of the force of gravitation and the centrifugal force arising from this rotation and the use of an earthbound rotating frame of reference. It is directed normal to sea level and to its geopotential surfaces." 11 To limit confusion, this document uses "gravitation" when it refers only to the attractive force between two or more masses and uses "gravity" to represent free-fall acceleration measured in a world-fixed reference frame. Equating gravity and gravitation is understandable. Centrifugal acceleration is very small compared to gravitation on the Earth. At the equator where centrifugal acceleration is greatest, the centrifugal acceleration is $\sim 0.35 \%$ the size of the gravitational force. One would have to ascend 11 kilometers $(\sim 36,000 \mathrm{ft})$ to experience a similar change in gravitation. Nevertheless, one must remain cognizant of the difference between gravity and gravitation when the fidelity of one's models includes terms this small. Standard gravity has its roots in gravity measurements and incorporates the centrifugal acceleration due to the Earth's rotation. Also, the reference ellipsoid (see section II) is defined as an equipotential surface of the potential of gravity. The centrifugal acceleration is what causes the equipotential surface to be an ellipsoid. If the world did not rotate, only gravitation would define the equipotential surface; that surface would be a sphere.

The rotating reference frame is an important part of the definition of gravity. From the perspective of an inertial observer, the world only imparts centrifugal acceleration on objects that rest on its surface. Objects that float above the surface experience only gravitation, though an object that originated on the surface will continue to carry the moment imparted to it by the world (and therefore won't immediately fly backward once it is free of the surface). Though the inertial observer sees only gravitation on the floating object, a world-fixed observer will see a combination of gravitation and a centrifugal acceleration that is a consequence of the observer rotating with the world. The distinction is important when matching observed free-fall in non-rotating world models (see section IV).

\section{B. Gravity and Gravitation Models}

This paper will investigate the interaction of four gravity/gravitation models with the three world models. The gravity/gravitation models are: constant gravity or gravitation, free-air reduction, Newtonian gravitation of a point mass, and a spherical harmonic expansion of the gravitational potential. The models are discussed in order of increasing fidelity and computation. The paper is concerned with how gravitation models of the world as a whole interact with the world model. These gravitation models are static in world fixed-coordinates. Time-varying perturbations such as third-body gravitation and tidal perturbations are not included in this investigation. The formulas in this section assume that gravity has a negative sense and that centrifugal acceleration is positive.

\section{Constant Gravity and Constant Gravitation}

The constant gravity model sets gravity to a constant value that does not change with position relative to the world. However, theoretical gravity on the reference ellipsoid differs with geodetic latitude. Between the equator $\left(-9.7803253359 \mathrm{~m} / \mathrm{s}^{2}\right)$ and the pole $\left(-9.8321849378 \mathrm{~m} / \mathrm{s}^{2}\right)$, gravity differs by $0.529 \%$ (or $\left.0.052 \mathrm{~m} / \mathrm{s}^{2}\right)^{1}$. Centrifugal acceleration imparted by the Earth's rotation accounts for the majority of the difference. It is $\sim 0.35 \%$ of gravitation at the equator and decreases to zero at the pole. The ellipsoidal distribution of mass and decreasing geocentric radius as one travels from the equator to the pole accounts for the remainder of the difference. For the Earth, standard gravity $\left(-9.80665 \mathrm{~m} / \mathrm{s}^{2}\right)$ is frequently used as the constant for this model. As stated in section III.A, standard gravity was defined as a unit, not a modeling parameter. An alternative constant is the mean gravity on the WGS84 ellipsoid, $-9.7976432222 \mathrm{~m} / \mathrm{s}^{2}$. One can also use the theoretical gravity for the geodetic latitude of the region in which the simulation is run, if the region is small. A regional gravity constant will be called an origin-based constant. The closed form equation for gravity on the surface of an ellipse is: ${ }^{1}$

$$
g^{s}=g_{e}^{s} \frac{1+k \sin ^{2} \phi}{\sqrt{1-e^{2} \sin ^{2} \phi}}
$$

$\mathrm{g}^{\mathrm{s}}$ is the surface gravity at geodetic latitude $\phi . \mathrm{g}_{\mathrm{e}}^{\mathrm{s}}$ is surface gravity at the equator. e is the eccentricity of the ellipse. $\mathrm{k}$ is given by the equation: 


$$
k=\frac{b g_{p}^{s}}{a g_{e}^{s}}-1
$$

$\mathrm{a}$ and $\mathrm{b}$ are the semi-major and semi-minor axes of the ellipse, respectively. $\mathrm{g}_{\mathrm{p}}^{\mathrm{s}}$ is the surface gravity at the pole. Equation 1 is called the Gravity Formula. Substituting values for the Earth, the Gravity Formula becomes:

$$
g^{s}=-9.7803253359 \frac{1+0.00193185265241 \sin ^{2} \phi}{\sqrt{1-0.00669437999014 \sin ^{2} \phi}} \mathrm{m} / \mathrm{s}^{2}
$$

The 1980 Geodetic Reference System provides a computationally less expensive approximation to the gravity on the ellipsoid called the International Gravity Formula: ${ }^{2,12}$

$$
\begin{aligned}
& g^{s}=-9.780327\left(1+0.0053024 \sin ^{2} \phi-0.0000058 \sin ^{2} 2 \phi\right) \mathrm{m} / \mathrm{s}^{2} \\
& =-9.780327\left(1+\sin ^{2} \phi\left(0.0052792+0.0000232 \sin ^{2} \phi\right)\right) \mathrm{m} / \mathrm{s}^{2}
\end{aligned}
$$

The first equation is the original representation in the 1980 International Gravity Formula. The second equation is an alternative form that requires the computation of only one transcendental function.

The above constants and formula are gravity-based and include a component of centrifugal acceleration. One can also create a constant model for gravitation. One option is to subtract the centrifugal acceleration at a given geodetic latitude from the gravity at the same latitude. Equation 5 gives the component of centrifugal acceleration that is normal to the reference ellipsoid $\left(\mathrm{g}_{\mathrm{c}}^{\mathrm{s}}\right)$ at geodetic latitude $(\phi)$ :

$$
g_{c}^{s}=\omega^{2} \frac{a \cos ^{2} \phi}{\sqrt{1-e^{2} \sin ^{2} \phi}}=\frac{0.033915706 \cos ^{2} \phi}{\sqrt{1-0.00669437999014 \sin ^{2} \phi}} \mathrm{m} / \mathrm{s}^{2}
$$

$\omega$ is the angular velocity of the Earth. The second formulation of Equation 5 inserts the corresponding values from the WGS84 Earth model. ${ }^{1}$ Options for global gravitation constants include the gravitation component of standard gravity, the mean gravitation over the surface of the reference ellipsoid, or computing a gravitation constant in the locality of the simulation using Newton's universal law of gravitation for a point mass (Equation 7). To compute the gravitation component of a gravity constant, one first determines the geodetic latitude that computes equal gravity in the Gravity Formula. This geodetic latitude will be called the equivalent latitude of the gravity constant. For standard gravity, the equivalent latitude is $\pm 45.5^{\circ}$. The equivalent latitude of mean gravity is $\pm 35.4^{\circ}$. The latitude can then be entered into Equation 5 to compute the centrifugal acceleration component of the gravity constant. The centrifugal acceleration component is subtracted from the gravity constant to reveal the gravitation component.

\section{Free-Air Reduction}

The constant gravity and gravitation models do well near the surface of the world, but they fail to model the behavior of gravity and gravitation as one moves away from the surface. The free-air reduction in geodesy is an option for modeling the change of gravity with height within the vicinity of the surface. The free-air reduction is a linear approximation for the vertical gradient of gravity in the neighborhood of the reference geoid; the linear change in gravity with height is approximately $-3.086 \times 10^{-6} \mathrm{~s}^{-2}{ }^{3}$ The resulting gravity formula is:

$$
g=g^{s}+3.086 \times 10^{-6} h
$$

where $\mathrm{h}$ is the height above the surface. The free air reduction provides a good approximation of gravity within altitudes traveled by subsonic aircraft. At $12.2 \mathrm{~km}(\sim 40,000 \mathrm{ft})$, the free air reduction differs from theoretical gravity by less than $1.5 \times 10^{-4} \mathrm{~m} / \mathrm{s}^{2}$. A similar formula can be used to simulate changes in gravitation by removing the centrifugal acceleration component of the linear constant; the resulting constant is $3.082 \times 10^{-6} \mathrm{~s}^{-2}$.

\section{Newton's Universal Law of Gravitation for a Point Mass}

According to Newton's universal law of gravitation, the attracting force between two masses is inversely proportional to the square of the distance between them and directly proportional to the product of their masses. The gravitation exerted by a world on an object depends on the distribution of its mass. A spherically symmetric mass is a good first approximation. A spherically symmetric mass does not require that the density of the world be constant; density can change, but only along the radius from the world's center of mass. For example, geology describes the Earth as consisting of layers (inner core, outer core, mantle, etc.) that are roughly concentric spheres. Though the density of each layer differs, they differ along a radius from the center. A spherically symmetric mass can be treated as if all of its mass where located at its center, i.e. a point mass. One can compute the gravitational acceleration imparted by spherically symmetric world using Equation 7:

$$
\vec{\gamma}=-\frac{G M}{|\mathbf{r}|^{2}} \hat{\mathbf{r}}
$$


where

$\vec{\gamma}$

$\mathrm{G}$

is the gravitational acceleration vector

M

$|\mathbf{r}|$

is the universal constant of gravitation, $6.673 \times 10^{-11} \mathrm{~m}^{3} / \mathrm{kg} \mathrm{s}^{2}$.

is the mass of the world.

$\hat{\mathbf{r}}$

is the magnitude of the position vector from the object to the center of the world.

is the corresponding unit vector.

The formula is valid for $\vec{r}$ greater than the radius of the world. The product of $\mathrm{G}$ and $\mathrm{M}$ is constant for a world and is called the gravitational constant for the world. It is usually given the symbol $\mu$. The Earth's gravitational constant is $3.986004418 \times 10^{14} \mathrm{~m}^{3} / \mathrm{s}^{2}$. This constant includes the atmosphere. The atmosphere exerts gravitational attraction on objects outside the atmosphere but not on objects on the world's surface (assuming that the world and its atmosphere are spherically symmetric). Items traveling through the atmosphere would experience the gravitational attraction of the atmosphere at lower altitudes, but not at higher altitudes. Fortunately, the Earth's atmosphere is a small fraction of its total mass. The gravitational constant of the atmosphere is $3.5 \times 10^{8} \mathrm{~m}^{3} / \mathrm{s}^{2}$, less than $10^{-6}$ the size of the Earth's gravitational constant. ${ }^{1}$ Simulations can use one gravitational constant for use within and outside the atmosphere, and this will introduce very little error to gravitation calculations within the atmosphere.

Newton's law computes only gravitation, not world-fixed gravity. Gravity requires the inclusion of the centrifugal acceleration imparted by the world's rotation. Though gravitation decreases with the square of the distance between the center of mass of the world and the object, centrifugal acceleration increases with distance:

$$
g \propto O\left(-\frac{G M}{r^{2}}\right)+O\left(\omega^{2} r\right)
$$

A gravity formula that obeys Equation 8 can be developed by separating the centrifugal acceleration at the surface $\left(\mathrm{g}_{\mathrm{c}}^{\mathrm{s}}\right)$ from surface gravity $\left(\mathrm{g}^{\mathrm{s}}\right)$ :

$$
g=\left(g^{s}-g_{c}^{s}\right)\left(\frac{R}{R+h}\right)^{2}+g_{c}^{s}+\omega^{2} h \cos ^{2} \phi
$$

where $\mathrm{R}$ is a geodetic radius that is a function of $\mathrm{g}^{\mathrm{s}}$. For the Earth, the Gravity Formula (Equation 1 or 4 ) defines $\mathrm{g}^{\mathrm{s}}$. Equation 5 defines $\mathrm{g}_{\mathrm{c}}^{\mathrm{s}}$. On Earth, gravitation is more than $300 \%$ larger than centrifugal acceleration near the surface; assuming that centrifugal acceleration is negligible, equation 9 reduces to:

$$
g=g^{s}\left(\frac{R}{R+h}\right)^{2}
$$

Equation 10 has the form of a point-mass model for gravity. The value for the radius remains a question. The underlying physical representation of equation 10 is a spherical equipotential surface of gravity. Thus, a spherically symmetric world is used to determine the radius. For origin-based gravity constants, the desired radius produces the same gravity at the equivalent latitude of the constant. The radius is the solution to the following equation:

$$
g^{s}+\frac{G M}{R^{2}}-\omega^{2} R \cos ^{2} \phi=0
$$

For global constants, a sphere whose mean gravity equals the constant provides the radius. This radius is computed from Equation 11 by setting $\cos ^{2} \phi$ equal to $2 / 3$. For standard gravity, the radius is $6368090.9 \mathrm{~m}$. Reasonable choices for radius have minor impact on computed gravity. For example, if the equatorial radius were used instead of $6368090.9 \mathrm{~m}$ for the standard gravity constant, the difference in computed gravity at $30.5 \mathrm{~km}(100,000 \mathrm{ft})$ would be $7.3 \times 10^{-5} \mathrm{~m} / \mathrm{s}^{2}$. Equation 11 defines a constant radius for a given $\mathrm{g}_{\mathrm{s}}$. Thus, point-mass gravity becomes a function of height only and its value can be applied along the surface normal, regardless of how the surface is modeled.

The point-mass gravity equation does not, however, provide better fidelity than the free air reduction at nearsurface altitudes traveled in non-rotating models of the Earth. The mean error of both models relative to the equipotential ellipsoid were computed and compared; origin-based gravity constants were used to isolate the differences in modeling gravity changes with height. At $12.2 \mathrm{~km}(40,000 \mathrm{ft})$, the mean error of the free-air reduction and pointmass gravity are $1.038 \times 10^{-4}$ and $1.362 \times 10^{-4} \mathrm{~m} / \mathrm{s}^{2}$ respectively. The error of the two models is equal at $\sim 15.9 \mathrm{~km}$ $(\sim 52,000 \mathrm{ft})$; above this height, point-mass gravity provides lower errors. Pairing either model with a non-rotating world will produce equivalent gravity results near the surface. The point-mass gravity model contains more mathematical operations than the free air reduction. Thus, the free-air reduction emerges as the best solution for changes of gravity with height near the surface. This paper will not include the point-mass gravity model in its analysis.

4. Spherical Harmonic Expansion of the Gravitational Potential

Most worlds are not spherically symmetric masses. The Earth, for example, bulges at the equator as a consequence of its rotation. The density of the Earth also varies from location to location. The landmass topography and 
the oceans are visible reminders that the Earth is not a symmetric mass. The variability of the Earth's mass is modeled by expressing the world's gravitational potential as a spherical harmonic expansion. ${ }^{\S}$ Even amongst geodesists, the gravitational potential models are also loosely referred to as "geopotential models" though "geopotential" is otherwise defined as the potential arising from gravity, i.e. gravitation plus centrifugal force due to the world's rotation. ${ }^{11}$ The spherical harmonic expansion of gravitational potential has the form: ${ }^{1,3,13}$

$$
V\left(r, \phi_{g}, \lambda\right)=\frac{G M}{r}\left(1+\sum_{n=2}^{\infty} \sum_{m=0}^{n}\left(\frac{a}{r}\right)^{n} P_{n m}\left(\sin \phi_{g}\right)\left[C_{n m} \cos (m \lambda)+S_{n m} \sin (m \lambda)\right]\right)
$$

where

$\mathrm{V}$ is the gravitational potential at geocentric spherical coordinates: radius $(\mathrm{r})$, latitude $\left(\phi_{\mathrm{g}}\right)$, and longi-

tude $(\lambda)$

$\phi_{\mathrm{g}} \quad$ is the geocentric latitude

$\mathrm{r} \quad$ is the geocentric radius

$\lambda \quad$ is the geocentric longitude

$\mathrm{G} \quad$ is the universal gravitational constant

$\mathrm{M} \quad$ is the mass of the world

a is the semi-major axis of the reference ellipsoid

$\mathrm{n} \quad$ is the degree of the harmonic term

$\mathrm{m} \quad$ is the order of the harmonic term

$\mathrm{C}_{\mathrm{nm}}, \mathrm{S}_{\mathrm{nm}}$ are the harmonic coefficients

$\mathrm{P}_{\mathrm{nm}}\left(\sin \phi_{\mathrm{g}}\right)$ is the associated Legendre polynomial of order $\mathrm{n}$ and degree $\mathrm{m}$ with an argument of $\sin \left(\phi_{\mathrm{g}}\right)$. See Ref. 3 for a definition of the Legendre polynomials.

A given "geopotential model" defines the world's gravitational constant (GM), the semi-major axis of the reference ellipse (a), and the harmonic coefficients $\left(\mathrm{C}_{\mathrm{nm}}\right.$ and $\left.\mathrm{S}_{\mathrm{nm}}\right)$. These constants are derived empirically using a combination of surface gravity data, satellite altimeter data, and satellite tracking data. Geopotential models in common use include: ${ }^{13,14}$

- GEM (Goddard Earth Model) developed by the NASA Goddard Space Flight Center

- TEG (Texas Earth Gravity) developed by the University of Texas (UT)

- OSU-91A (Ohio State University) developed by Ohio State University (OSU)

- GRIM4 developed by GeoForschungs-Zentrum Potsdam (GFZ, Germany) and Groupe de Geodesie Spatiale (GRGS, France)

- JGM (Joint Gravitational Model) developed by NASA, UT, OSU, and the Centre Natiornal d'Etudes Spatiales (CNES)

- EGM96 (Earth Gravitational Model) developed by NASA, OSU, and the National Imagery and Mapping Agency (NIMA)

Published geopotential models list normalized harmonic coefficients, which must be used with normalized Legendre polynomials. Normalization reduces the range of magnitudes for $\mathrm{P}_{n m}, \mathrm{C}_{\mathrm{nm}}$, and $\mathrm{S}_{\mathrm{nm}}$ to prevent numerical overflow or underflow on computers. ${ }^{15}$ Ref. 1 provides the normalization equations for $\mathrm{P}_{\mathrm{nm}}, \mathrm{C}_{\mathrm{nm}}$, and $\mathrm{S}_{\mathrm{nm}}$. The spherical harmonic expansion has the favorable property that it may be truncated at any point without the need to compute new harmonic coefficients. If a geopotential model publishes coefficients to degree and order 70 , one can create a valid gravitation computation using only the coefficients to degree and order 36.

One obtains the gravitational acceleration by taking the gradient of the gravitational potential. Taking the gradient of Equation 12 in spherical coordinates results in an equation with a singularity at the poles. Pines developed an alternate representation that removes the singularity and preserves the harmonic coefficients. ${ }^{15,16}$ There exist a variety of formulations of Pines representation for use in computing gravitation from a geopotential model. The reader is directed to references 15 and 16 for a complete treatment on computing gravitation from a geopotential model. The subject cannot easily be summarized for incorporation into this paper.

$\S$ A spherical harmonic expansion satisfies Laplace's equation in spherical coordinates, and functions that satisfy Laplace's equation are said to be harmonic.

" This paper defines geocentric latitude as it is used in the WGS84 model. Geocentric latitude is the angle between the equatorial plane and the radius from the center of the world to a point. The point may be on or above the surface of the reference ellipsoid. 
The full geopotential model provides gravitation values appropriate for high accuracy orbit simulation. Ignoring order higher than zero (i.e. $\mathrm{m}=0$ ) greatly simplifies Equation 12:

$$
V\left(r, \phi_{g}\right)=\frac{G M}{r}\left(1+\sum_{n=2}^{\infty}\left(\frac{a}{r}\right)^{n} C_{n} P_{n}\left(\sin \phi_{g}\right)\right)
$$

Where $\mathrm{C}_{\mathrm{n}}=\mathrm{C}_{\mathrm{n}, 0}$ and $\mathrm{P}_{\mathrm{n}}\left(\sin \phi_{\mathrm{g}}\right)=\mathrm{P}_{\mathrm{n} 0}\left(\sin \phi_{\mathrm{g}}\right)$. The harmonic terms where $\mathrm{m}=0$ are called zonal harmonics. The zonal harmonics capture mass distribution effects which do not depend on longitude, i.e. the effect is symmetric about the North-South axis. Visualize it as taking slices of the earth along its lines of latitude. When $\mathrm{n}$ is even, the effect is symmetric about the equatorial plane; and, when $\mathrm{n}$ is odd, the effect is asymmetric., ${ }^{3,17}$ The harmonics that disappeared from the general equation (Equation 12) are divided into sectorial harmonics $(n=m)$ and tesseral harmonics $(\mathrm{n} \neq \mathrm{m})$. Sectorial harmonics capture mass distributions effects that depend only on longitude; think of it as dividing the world into sections like an orange. Tesseral harmonics capture mass distributions effects that vary with both latitude and longitude, similar to dividing the world into a checkerboard. Though less accurate than Equation 12, Equation 13 remains an improvement over the point-mass gravitation model. One advantage to Equation 13 is that its gradient doesn't introduce a singularity at the poles. The gradient term that introduces the singularity in Equation 12 contains $\partial \mathrm{V} / \partial \lambda$, which equals zero for Equation 13. Taking the gradient in Cartesian coordinates results in the following gravitation equations to degree four: ${ }^{17}$

$$
\begin{aligned}
& \ddot{x}=-\mu \frac{x}{r^{3}}\left[1+\frac{3 C_{2} a^{2}\left(5 z^{2}-r^{2}\right)}{2 r^{4}}-\frac{5 C_{3} a^{3} z\left(3 r^{2}-7 z^{2}\right)}{2 r^{6}}+\frac{5 C_{4} a^{4}\left(3 r^{4}-42 z^{2} r^{2}+63 z^{4}\right)}{8 r^{8}}\right] \\
& \ddot{y}=\ddot{x} \frac{y}{x} \\
& \ddot{z}=-\mu \frac{z}{r^{3}}\left[1-\frac{3 C_{2} a^{2}\left(3 r^{2}-5 z^{2}\right)}{2 r^{4}}-\frac{C_{3} a^{3}\left(30 z^{2} r^{2}-35 z^{4}-3 r^{4}\right)}{2 z r^{6}}+\frac{5 C_{4} a^{4}\left(15 r^{4}-70 z^{2} r^{2}+63 z^{4}\right)}{8 r^{8}}\right]
\end{aligned}
$$

where

$$
\begin{array}{ll}
\mathrm{x}, \mathrm{y}, \mathrm{z} & \text { are the world-fixed coordinates of the object } \\
\mathrm{r} & \text { is the magnitude of the geocentric radius } \\
\mathrm{C}_{2} & =-1.08262668355 \mathrm{E}-3 \\
\mathrm{C}_{3} & =+2.53265648533 \mathrm{E}-6 \\
\mathrm{C}_{4} & =+1.61962159136 \mathrm{E}-6 \\
\mathrm{a} & \text { is the semi-major axis of the world } \\
\mu & \text { is the gravitational constant for the world (G M). }
\end{array}
$$

The values for the zonal harmonics were taken from Ref. 14 and multiplied by the normalization function $\sqrt{2 n+1}$. Ref. 17 shows the derivation of Equations 14 through 16 and provides these equations to degree six. ${ }^{\#}$ However, Ref. 17 uses a formulation for Equation 13 that gives a negative sign to the summation term. Equations 14 through 16 use a positive summation term so that they can use published zonal harmonic coefficients $\left(C_{n, 0}\right)$ without change of sign. In Ref. 17, the zonal harmonic coefficients are given the symbol $\mathrm{J}_{\mathrm{n}}$ and are the negative of the $\mathrm{C}_{\mathrm{n}, 0}$ coefficients. Zonal harmonic coefficients published as $\mathrm{J}_{\mathrm{n}}$ will also be of opposite sign. (GRS80 uses $\mathrm{J}_{2}$ as one of the parameters to define its equipotential ellipsoid for the Earth, and its value of $J_{2}$ is widely published. ${ }^{3}$ )

To analyze the interaction of the geopotential model with world models, this paper uses a simplified geopotential model that approximates the idealized gravitation field of the equipotential ellipsoid. The sectorial and tesseral harmonic coefficients reduce to zero because the gravitational field of the equipotential ellipsoid has rotational symmetry about the pole, and the odd zonal harmonics reduce to zero because the gravitational field is symmetric about the equatorial plane. ${ }^{3}$ Only the even zonal harmonics remain to describe the field. Moritz shows that the second degree zonal harmonic $\left(\mathrm{C}_{2,0}\right)$ is then a function of the world's mass, equatorial radius, and moments of inertia (Equation 17). ${ }^{2,3}$ All subsequent zonal harmonics are functions of $\mathrm{C}_{2,0}$ (Equation 18).

$$
C_{2,0}=\frac{I_{z z}-I_{x x}}{M a^{2}} \quad, \quad C_{2 n, 0}=(-1)^{n} \frac{3 e^{2 n}}{(2 n+1)(2 n+3)}\left(1-n-5 n \frac{C_{2,0}}{e^{2}}\right)
$$

\footnotetext{
\# Note that the equation for $\ddot{Z}$ in reference 17 has the exponent for the $(\mathrm{a} / \mathrm{r})$ term swapped for $\mathrm{J}_{2}$ and $\mathrm{J}_{3}$.
} 
Since the world's mass properties cannot be measured directly, $\mathrm{C}_{2,0}$ is empirically derived. For the Earth, the first two even zonal harmonics $\left(\mathrm{C}_{2,0}\right.$ and $\left.\mathrm{C}_{4,0}\right)$ provide gravitation accuracy to six significant digits. Equation 19 is the resulting geopotential equation.

$$
V\left(r, \phi_{g}\right)=\frac{\mu}{r}\left[1+\left(\frac{a}{r}\right)^{2} C_{2,0} \frac{\left(3 \sin ^{2} \phi_{g}-1\right)}{2}+\left(\frac{a}{r}\right)^{4} C_{4,0} \frac{\left(35 \sin ^{4} \phi_{g}-30 \sin ^{2} \phi_{g}+3\right)}{8}\right]
$$

Equations 20 and 21 are the gravitation equations that are the gradient of Equation 19.

$$
\begin{aligned}
& \gamma_{r}=\frac{\partial V}{\partial r}=-\frac{\mu}{r^{2}}\left[1+\frac{3}{2}\left(\frac{a}{r}\right)^{2} C_{2,0}\left(3 \sin ^{2} \phi_{g}-1\right)+\frac{5}{8}\left(\frac{a}{r}\right)^{4} C_{4,0}\left(35 \sin ^{4} \phi_{g}-30 \sin ^{2} \phi_{g}+3\right)\right] \\
& \gamma_{\phi_{g}}=\frac{1}{r} \frac{\partial V}{\partial \phi_{g}}=\cos \phi_{g} \sin \phi_{g} \frac{\mu}{r^{2}}\left(\frac{a}{r}\right)^{2}\left[3 C_{2,0}+\frac{1}{2}\left(\frac{a}{r}\right)^{2} C_{4,0}\left(35 \sin ^{2} \phi_{g}-15\right)\right]
\end{aligned}
$$

For the Earth, $\mathrm{C}_{2,0}=-0.00108262982131$ and is computed from the WGS84 normalized coefficient ${ }^{1} ; \mathrm{C}_{4,0}=$ $2.37091120053 \mathrm{E}-06$ and is derived using Equation 18. The paper's results use terms to degree eight because the first four even zonal harmonics are required to match the WGS84 published values to all ten significant digits.

\section{Pairing World and Gravity/Gravitation Models}

The paper will refer to the combination of a gravity/gravitation model with a world model as a gravitation-world pair. Some gravitation-world pairs generate side effects that depart from reality. The side effects represent very small errors in gravity but present challenges to applications requiring high accuracy. Increases in computing power now allow engineers to select higher fidelity at little additional cost to runtime. Increasing the fidelity of the world model and the gravitation model independently does not necessarily lead to an overall increase in fidelity for near surface operations. The pair may emit errors that overshadow the increased fidelity of each individual model. This investigation will use the equipotential ellipsoid as the truth state against which the error of gravitation-world pairs are measured. The error of ignoring high fidelity effects acts as a benchmark for examining the effective fidelity of gravitation-world combinations. This paper will present errors as percentages of the mean gravity. On the Earth, the geopotential model introduces spherical harmonic modeling of the Earth's non-spherical mass distribution and reduces gravitation prediction errors below $0.328 \%$ (mean error $=0.126 \%$ ) relative to point-mass modeling of gravitation. Higher fidelity world models introduce the centrifugal acceleration term whose absence represents an error as large as $0.346 \%$ of gravity on the surface (mean error $=0.231 \%$ ). Errors from the two effects are not additive; they partly counteract at points over the globe. On the reference ellipsoid, ignoring both effects leads to maximum and mean gravity errors of $0.328 \%$ and $0.231 \%$, respectively. This is the error of modeling point-mass gravitation only and will be referred to as the minimum fidelity. At heights above the surface, the errors of modeling only point-mass gravitation will continue to define the minimum fidelity. A gravitation-world pair will fail to meet the minimum fidelity if it exceeds either the maximum or mean error. A good gravitation-world pair also exhibits greater fidelity than pairs using lesser fidelity models. This section explores the results of combining the four gravity/gravitation models with each of the three world models.

\section{A. Gravity Models in Combination with the Flat World}

The flat world does not have a center of mass. Instead, the surface plane and any plane parallel to the surface are modeled as an equipotential surface of the gravity potential. The gravity vector always intercepts the flat surface at $90^{\circ}$. The flat world does not rotate. Centrifugal acceleration due to the world's rotation will, therefore, not appear as term in the motion of objects relative to the world. To faithfully model the free-fall acceleration that is observed on the surface of the world, the simulation should model gravity, not gravitation.

For the constant gravity model, the gravity constant determines the range of error introduced by the model. The simulation may use a global constant for the model or origin-based constants. Though it is defined as a unit and not a property of the Earth, standard gravity is a frequently used global constant for modeling Earth's gravity; the mean gravity of the Earth is another candidate. Any global constant matches the theoretical gravity at the constant's equivalent latitude. The global constant yields errors at other locations. The equivalent latitude of standard gravity is $\pm 45.5^{\circ}$; the equivalent latitude of the mean gravity is $\pm 35.6^{\circ}$. Table 1 shows the errors of the flat-world, constantgravity pair with standard gravity and mean gravity as the constants. The mean error is taken over the surface of the reference ellipsoid, treating the flat world as a locally flat plane on an ellipsoid. If the mean were taken over a plane with equidistant lines of latitude, the mean error would be $0.01651 \mathrm{~m} / \mathrm{s}^{2}(0.169 \%)$ for standard gravity and 0.01741 $\mathrm{m} / \mathrm{s}^{2}(0.178 \%)$ for mean gravity. Though the mean gravity produces a lower mean error than standard gravity, its 
maximum error is larger and exceeds the minimum fidelity. By this criterion, standard gravity is the better global constant and is used for the remainder of the analysis. (The optimal gravity constant would be $-9.80005 \mathrm{~m} / \mathrm{s}^{2}$ ! It's maximum and mean errors would be $-0.321 \%$ and $0.141 \%$ respectively.)

Table 1 Errors of the Flat World with Constant Gravity

\begin{tabular}{|l|r|r|r|r|}
\cline { 2 - 5 } \multicolumn{1}{c|}{} & \multicolumn{2}{c|}{ Standard Gravity } & \multicolumn{2}{c|}{ Mean Gravity } \\
\cline { 2 - 5 } \multicolumn{1}{c|}{} & Absolute Error $\left(\mathrm{m} / \mathrm{s}^{2}\right)$ & Percentage Error & Absolute Error $\left(\mathrm{m} / \mathrm{s}^{2}\right)$ & Percentage Error \\
\hline Equatorial Gravity & -0.02632 & $-0.268 \%$ & -0.01732 & $-0.176 \%$ \\
\hline Polar Gravity & 0.02553 & $0.261 \%$ & 0.03454 & $0.353 \%$ \\
\hline Mean Error & 0.01596 & $0.163 \%$ & 0.01132 & $0.136 \%$ \\
\hline
\end{tabular}

The gravity constant includes a component of constant centrifugal acceleration and a component of constant gravitation. Therefore, the pair is expected to provide some fidelity improvement over ignoring centrifugal acceleration, but should be worse than ignoring the non-spherical distribution of the Earth's mass because the gravitation component is constant over the surface. The mean error is less than $60 \%$ of the mean error for ignoring the centrifugal acceleration $(0.231 \%)$ and lies above the mean error of ignoring the non-spherical distribution of the Earth's mass $(0.126 \%)$ The flat-world, constant-gravity model does generate fidelity inline with its modeling, retaining some fidelity improvement for modeling a constant centrifugal acceleration. Origin-based gravity constants, defined by the Gravity Formulas (Equations 1 or 4), can further improve fidelity by providing the theoretical gravity for the starting location of the simulation during initialization. Since flat world models are valid for short distances that approximate a plane on the world surface, errors in surface gravity from origin-based constants will be miniscule over the region of the simulation. Origin-based gravity constants provide the full fidelity of theoretical gravity on the surface of the region and should be preferred in flat world models. Nevertheless, it is often desirable to use a global constant in order to easily compare and analyze simulation results regardless of starting location. This convenience sacrifices fidelity but remains within the minimum fidelity with the right global constant.

As one travels away from the surface of the world, the acceleration due to gravity decreases. A constant gravity model will introduce errors for objects above the surface. When using standard gravity as a global constant, the constant gravity model will fall below minimum fidelity at altitudes above $1.9 \mathrm{~km}(6200 \mathrm{ft})$. (Maximum error is exceeded; mean error is exceeded at $3.8 \mathrm{~km}$.) The origin-based gravity constant falls below the minimum fidelity above $7.4 \mathrm{~km}(24,300 \mathrm{ft})$. (Mean error is exceeded; maximum error is exceeded at $10.4 \mathrm{~km}$.) Above these heights, modeling fidelity grows worse than modeling only point-mass gravitation. A model that changes gravity with height is necessary to prevent further degradation of the gravity error. The free air reduction (Equation 6) offers a low-cost, linear relationship that provides accurate gravity values at altitudes typically traveled over flat world models. The free air reduction is a function of height above the surface. Point-mass and geopotential models would require a contrived radius to the "gravitational center". When used with an origin-based gravitation constant, the free-air reduction computes gravity with accuracy nearly equal to modeling the equipotential ellipsoid; but the accuracy is restricted to travel within distances and altitudes where the flat world is valid. This model maintains minimum fidelity below a height of $\sim 82 \mathrm{~km}$ (Maximum error exceeded). This is well above the altitudes typically flown for regional travel. The computational cost of this accuracy is one multiplication and one subtraction during runtime. This leaves no compelling reason to consider the point-mass or geopotential model for use in a flat world and the combinations will not be investigated. The free air reduction best matches the simplicity and minimal computation embodied by the flat world model.

\section{B. Gravity/Gravitation Models in Combination with the Sphere World}

The sphere is a good first approximation for modeling the curvature of a world and allows the simulation to models the world's rotation. Since most worlds are not perfect spheres, there are several definitions of the world's radius that modelers can employ to define the spherical model. Each has their advantages and disadvantages. This paper will focus on the impact that radius choice has on gravity modeling. Though all gravity and gravitation models will be applied to the sphere world, the point-mass gravitation model is used to evaluate the candidate radii since it describes the theoretical gravitation of spherically symmetric world. The mean gravity $\left(\bar{g}^{s}\right)$ on the surface of a rotating sphere is:

$$
\bar{g}^{s}=-\frac{G M}{R^{2}}+\frac{2}{3} \omega^{2} R
$$

Table 2 shows the mean gravity resulting from several candidate radii. The equatorial radius produces a mean gravity that is $0.224 \%$ smaller than the equipotential ellipsoid; the average of the equatorial and polar radii produces mean gravity that is $0.112 \%$ larger. The mean, equal-surface-area, and equal-volume radii are all clustered closely 
together and produce an average gravity that is nearly equal to the reference ellipse; the error is approximately $\pm 1 \times 10^{-5} \mathrm{~m} / \mathrm{s}^{2}$. From the perspective of gravity modeling, any of the last three radii are a good start for modeling a sphere Earth. Each also presents another modeling advantage. The mean radius minimizes the modeling error of the geocentric radius to the surface. The radius of equal surface area minimizes the error of modeling the surface distance between any two points on the Earth. The radius of equal volume produces a sphere with the same average density as the reference ellipsoid. This paper uses the equal-surface-area radius for the sphere model of the Earth since it produces the smallest error in mean gravity.

Table 2 Mean Earth Gravity of Spherical Earth for Different Radii

\begin{tabular}{|l|r|r|r|}
\hline \multicolumn{1}{|c|}{ Radius } & Length $(\mathrm{m})$ & Mean Gravity $\left(\mathrm{m} / \mathrm{s}^{2}\right)$ & $\Delta \bar{g}^{s}$ (Sphere - Ellipsoid) $\left(\mathrm{m} / \mathrm{s}^{2}\right)$ \\
\hline Equatorial & 6378137.0 & -9.775666 & $2197.68615 \mathrm{E}-05$ \\
\hline Mean & 6371008.8 & -9.797630 & $1.36438 \mathrm{E}-05$ \\
\hline Surface Area Equal to Ellipse & 6371007.2 & -9.797634 & $0.87350 \mathrm{E}-05$ \\
\hline Volume Equal to Ellipse & 6371000.8 & -9.797654 & $-1.09894 \mathrm{E}-05$ \\
\hline Average of Equatorial and Polar Radii & 6367444.7 & -9.808639 & $-1099.56043 \mathrm{E}-05$ \\
\hline
\end{tabular}

The outcomes of gravitation-world pairs with a sphere model depend on whether the sphere model adds the world's rotation. Each gravity/gravitation model will be discussed in the context of a non-rotating and rotating sphere. This paper will assume that the equations of motion for an object include the centrifugal acceleration term when rotation is active and that including the term is a pretext of modeling accuracy to that level. With rotation disabled, one must utilize a gravity model to incorporate the centrifugal acceleration term.

Like the flat world, the non-rotating sphere must use a gravity constant in its constant model. Since the Earth's reference ellipsoid is nearly a sphere, the constant gravity model has the same errors in the spherical world as in the flat world. However, simulations in the non-rotating sphere are valid over long distances. The spherical model cannot rely on the simulation maintaining locality with the origin. Origin-based gravity constants no longer have a guaranteed accuracy advantage over global gravity constants, especially for long North-South routes. The nonrotating sphere should therefore use a global gravity constant as the default; origin-based gravity constants can remain a user selection for simulations that primarily move East-West and desire additional accuracy. The nonrotating sphere must therefore trade its improved surface fidelity over long distances for lower gravity fidelity that represents a modest improvement over ignoring the centrifugal acceleration.

The rotating sphere models the centrifugal acceleration term of gravity in its acceleration equations. Therefore, using one constant gravity model for both the rotating and non-rotating sphere will effectively exaggerate the effect of centrifugal acceleration in the rotating sphere. The rotating sphere requires a gravitation constant. On the Earth, centrifugal acceleration is the largest contributor to variation in gravity over the globe. The variation in gravitation is much smaller, gravitation at the equator is $-9.81424 \mathrm{~m} / \mathrm{s}^{2}$ and gravitation at the pole is $-9.83218 \mathrm{~m} / \mathrm{s}^{2}$. The difference is $0.01794 \mathrm{~m} / \mathrm{s}^{2}$ or $0.183 \%$, about one third the variation of gravity. Using a global gravitation constant with a rotating sphere should produce smaller gravity errors than the global gravity constant with the non-rotating sphere. Candidates for a global constant include the gravitation component of standard gravity $\left(-9.82334 \mathrm{~m} / \mathrm{s}^{2}\right)$, the mean gravitation $\left(-9.82023 \mathrm{~m} / \mathrm{s}^{2}\right)$, and the point-mass gravitation (Equation 7) on the surface of the sphere $(-9.82023$ $\mathrm{m} / \mathrm{s}^{2}$ ). The radius for the sphere was chosen to minimize the error in mean gravity and, consequently, also produces gravitation equal to the mean gravitation; thus, the remainder of the investigation will use $-9.82023 \mathrm{~m} / \mathrm{s}^{2}$ as the gravitation constant. The resulting gravity values on the rotating sphere vary from $-9.78635 \mathrm{~m} / \mathrm{s}^{2}$ at the equator to $-9.82023 \mathrm{~m} / \mathrm{s}^{2}$ at the pole. Table 3 displays the gravity errors of the rotating sphere with constant gravitation. The mean is computed over the surface of the reference ellipse by equating the geodetic latitude of the ellipse with the geodetic latitude of the sphere (whose geodetic and geocentric latitudes are equal). Since point-mass gravitation equals the global gravitation constant on the surface of the sphere, the errors in Table 3 also apply to the point-mass gravitation model with the rotating sphere.

Table 3 Surface Gravity Errors of Constant or Point-Mass Gravitation with the Rotating Sphere

\begin{tabular}{|l|r|r|}
\cline { 2 - 3 } \multicolumn{1}{c|}{} & Absolute Error $\left(\mathrm{m} / \mathrm{s}^{2}\right)$ & Percentage Error \\
\hline Equatorial Gravity & 0.00603 & $0.061 \%$ \\
\hline Polar Gravity & -0.01196 & $-0.122 \%$ \\
\hline Mean Error & 0.00461 & $0.047 \%$ \\
\hline
\end{tabular}

The $0.184 \%$ span in error between equator and pole is equivalent to the variation of surface gravitation (it differs by $4 \times 10^{-5} \mathrm{~m} / \mathrm{s}^{2}$ ) and verifies that the variation in surface gravitation is the dominant modeling error of the global gravitation constant with the rotating sphere. Errors in centrifugal acceleration are negligible. The mean error of the constant gravitation model with a rotating sphere is an improvement over the lower fidelity non-rotating sphere with globally constant gravity. The error is also one-fifth the mean error of neglecting the centrifugal acceleration term 
$(0.231 \%)$. The rotating sphere with globally constant gravitation preserves the fidelity of adding the centrifugal acceleration term. Given these errors, the rotating sphere has less cause to use an origin-based gravitation constant, even as a user selection. A global gravitation constant should satisfy most simulations that accept the lower fidelity of a constant model. The rotating sphere with constant gravitation exhibits a mean gravity error that is less than half the mean error of neglecting the non-spherical distribution of the earth's mass $(0.126 \%)$. The latter error is based on point-mass gravitation on the reference ellipsoid. The errors, therefore, indicate that constant gravitation will better reproduce surface gravity on the reference ellipsoid than using point-mass gravitation. This gravitation-world pair would appear to offer enhanced fidelity above the addition of the centrifugal acceleration term. However, the enhanced fidelity is restricted to the vicinity of the sphere surface whose location in inertial space can differ by up to $14 \mathrm{~km}$ compared to the reference ellipsoid and, as discussed later, this gravitation-world pair introduces a tangential gravity that is not present in the real world.

Like the flat-world, the non-rotating sphere will fail to meet the minimum fidelity at altitudes above $\sim 1.9 \mathrm{~km}$ $(\sim 6200 \mathrm{ft})$ when paired with a global gravity constant. The free-air reduction (Equation 6$)$ can be paired with the non-rotating world to provide gravity changes with height. It is an appropriate gravity model to use with the nonrotating sphere whose validity is limited to low-speed objects that tend to travel near the surface. It makes little sense to pair a non-rotating world model with a gravitation model like the point-mass gravitation model (Equation 7). The non-rotating world loses the centrifugal acceleration contribution to gravity with no savings in computation. Adding the necessary centrifugal acceleration requires the same equation that appears in the rotating world; the resulting gravity fidelity will not be different. Thus, the paper only investigates the gravitational models in combination with a rotating world model.

The rotating sphere with constant gravitation will fail to meet the minimum fidelity at altitudes above $7.4 \mathrm{~km}$ $(24,300 \mathrm{ft})$. (Mean error exceeded. Maximum error is exceeded above $8.5 \mathrm{~km}$.) Above this height, modeling gravitation changes with height are required to avoid further degradation of the gravity error. (The rotating sphere already models the change of centrifugal acceleration with height.) An equation like the free-air reduction (Equation 6) can compute gravitation with height, but the height multiplier must be changed to $3.0828 \times 10^{-6} \mathrm{~s}^{-2}$ (removing the centrifugal acceleration component of the multiplier). The free-air reduction will maintain the minimum fidelity to a height of $157 \mathrm{~km}$. (Maximum error exceeded. Mean error exceeded at $183 \mathrm{~km}$.) These are low-Earth orbit altitudes, and tolerance for gravity errors at these altitudes is typically lower than the minimum fidelity. At $157 \mathrm{~km}$, the mean error of the free-air reduction for gravitation is $0.01733 \mathrm{~m} / \mathrm{s}^{2}$. The next alternative is the point-mass gravitation model (Equation 7). Its mean error at $157 \mathrm{~km}$ is almost $75 \%$ lower, $0.00458 \mathrm{~m} / \mathrm{s}^{2}$. The mean error of the pointmass gravitation model is slightly lower at altitude than at the surface $\left(0.00461 \mathrm{~m} / \mathrm{s}^{2}\right)$. The error will continue to decrease with altitude because Earth's gravitation converges to a point mass as distance increases. Though the freeair reduction for gravitation generates reasonably accurate gravity for objects close to the surface, its error increases with altitude. The point-mass gravitation model will maintain small errors for both near-surface and orbital simulations. Given that the rotating spherical world adds substantial computation over simpler world models, preserving low computation in the gravitation model is less urgent. The point-mass gravitation model has greater applicability and modeling fidelity for a modest increase in mathematical operations over the free-air reduction. There is little reason to select the free-air reduction over the point-mass gravitation model when pairing them with a rotating sphere model.

The rotating sphere provides much improved gravity errors near the surface than the non-rotating sphere with a modest increase in computation. It seems natural to conclude that simulations should always model rotation with a spherical model. But, rotation also introduces a problem for near surface simulations. Since centrifugal acceleration is perpendicular to the rotation axis and not the surface, rotation introduces a remnant tangential gravity acceleration on the surface $\left(\mathrm{g}_{\mathrm{t}}^{\mathrm{s}}\right)$ that is directed toward the equator. If a simulated object in the Northern hemisphere faces EastWest and lacks a side-force friction model, it will slowly drift southward while resting on the surface of the rotating sphere. The mean of $g_{t}^{s}$ is half that of the normal component of centrifugal acceleration $\left(-1 / 3 \omega^{2} \mathrm{R}=-0.01129 \mathrm{~m} / \mathrm{s}^{2}\right)$. (All values of $\mathrm{g}_{\mathrm{t}}^{\mathrm{s}}$ are given a sign indicating their direction in the Northern hemisphere. The sign of the numbers would be opposite in the Southern hemisphere.) $\mathrm{g}_{\mathrm{t}}^{\mathrm{s}}$ is largest at $45^{\circ}$ latitude where it is $-1 / 2 \omega^{2} \mathrm{R}$ and diminishes to zero as one moves toward the equator or the pole. Resting bodies do not experience a non-zero $\mathrm{g}_{\mathrm{t}}^{\mathrm{s}}$ in the real world (discounting local gravity anomalies). The world's mass would also experience $\mathrm{g}_{\mathrm{t}}^{\mathrm{s}}$ and drift toward the equator creating a bulge. As the world's mass deforms, gravitation will also drift away from the surface normal until the world forms an ellipsoid where the tangential component of gravitation cancels out the tangential component of centrifugal acceleration at the surface; i.e. an equipotential ellipsoid of the gravity potential is formed. The root causes of $\mathrm{g}_{\mathrm{t}}^{\mathrm{s}}$ are the surface modeling error in the rotating sphere and the gravitation error of the point-mass model; later discussion will demonstrate that these are nearly equal. Modifying the constant or point-mass gravitation models to drive $g_{t}^{s}$ to 
zero will change behavior off the surface and should be avoided. If $g_{t}^{s}$ is a problem for surface operations, one can create a simple compensating side-force friction model:

$$
f_{y}=\frac{1}{2} \omega^{2} R \sin (2 \phi) \sin (\psi)
$$

where $\mathrm{f}_{\mathrm{y}}$ is the side force friction in body coordinates and $\psi$ is the true heading of the object; it is assumed that the rolling friction model will counteract the $\mathrm{x}$-body axis component of $\mathrm{g}_{\mathrm{t}}^{\mathrm{s}}$. Equation 23 increases the computation in conflict with the low computation properties of the constant gravity/gravitation model. Simulations using a constant model may prefer to disable rotation over using Equation 23 at the additional cost of losing the Coriolis acceleration. Therefore, low-fidelity simulation of low-speed objects is the one case where the non-rotating sphere may be better suited than the rotating sphere.

The geopotential model improves the fidelity of modeling gravitation and would appear to remove one source of error that causes a non-zero $\mathrm{g}_{\mathrm{t}}^{\mathrm{s}}$. However, the mean tangential gravitation of the geopotential model on the sphere is $-0.01065 \mathrm{~m} / \mathrm{s}^{2}$, the same direction and nearly the same magnitude as the mean tangential centrifugal acceleration! The equatorial bulge, caused by a greater concentration of mass at the equator, deflects the gravitation vector away from the geocentric radius, toward the equator. Since the geocentric radius is also the surface normal on the sphere, the deflection in the gravitation vector creates a tangential component of gravitation toward the equator. The result is a doubling of $\mathrm{g}_{\mathrm{t}}^{\mathrm{s}}$ on the spherical Earth. The geopotential model worsens rather than improve the problem of $\mathrm{g}_{\mathrm{t}}^{\mathrm{s}}$ on the sphere. The geopotential model with the rotating sphere isolates the impact of surface modeling error on $\mathbf{g}_{\mathrm{t}}^{\mathrm{s}}$. In the next section, the rotating ellipsoid with point mass-gravitation will isolate the impact of gravitation modeling errors on $\mathrm{g}_{\mathrm{t}}^{\mathrm{s}}$. It should produce a counteracting tangential acceleration of gravitation that points toward the pole and has a mean of $\sim 0.02194 \mathrm{~m} / \mathrm{s}^{2}$.

Combining a geopotential model with a sphere model worsens the fidelity in modeling $\mathrm{g}_{\mathrm{t}}^{\mathrm{s}}$. The next question is whether the error is a tradeoff for improved fidelity of the normal surface gravity $\left(\mathrm{g}_{\mathrm{n}}^{\mathrm{s}}\right)$ or whether fidelity of $\mathrm{g}_{\mathrm{n}}^{\mathrm{s}}$ also worsens. When computing the mean gravitation of Equation 20, the zonal harmonic terms all produce a mean of zero over the sphere. Thus, the mean gravitation $\left(-9.82023 \mathrm{~m} / \mathrm{s}^{2}\right)$ of Equation 20 equals the mean gravitation of the sphere. The mean gravity $\left(-9.79764 \mathrm{~m} / \mathrm{s}^{2}\right)$ is also the same. Like the rotating sphere with point-mass gravitation, the values agree with the equipotential ellipse. The computed gravity at the equator is $-9.80238 \mathrm{~m} / \mathrm{s}^{2}$ and gravity at the pole is $-9.78838 \mathrm{~m} / \mathrm{s}^{2}$. In opposition to the real world, the largest gravity exists at the equator, not the pole! The variation of modeled gravity over the surface is however is small, $0.01400 \mathrm{~m} / \mathrm{s}^{2}(0.143 \%)$. Table 4 shows the resulting surface gravity errors. The span of the error is $0.06586 \mathrm{~m} / \mathrm{s}^{2}(0.672 \%)$. The error span exceeds the actual variation of gravity on the surface of the Earth $(0.529 \%)$ and is the sum of this actual variation and the modeled variation. The modeled variation, therefore, quantifies the worsening fidelity of the near-surface gravity that the geopotential model brings to the sphere world. Though the geopotential model improves gravitation fidelity, it fails to meet the minimum fidelity when combined with a rotating sphere. (Maximum error is exceeded.) It also produces maximum and mean errors that are worse than the point-mass gravitation model with the rotating sphere. Simulations configured for a sphere world would be better off reverting to a point-mass gravitation model.

Table 4 Surface Gravity Errors of the Geopotential Model with the Rotating Sphere

\begin{tabular}{|l|r|r|}
\cline { 2 - 3 } \multicolumn{1}{c|}{} & Absolute Error $\left(\mathrm{m} / \mathrm{s}^{2}\right)$ & Percentage Error \\
\hline Equatorial Gravity & -0.02205 & $-0.225 \%$ \\
\hline Polar Gravity & 0.04381 & $0.447 \%$ \\
\hline Mean Error & 0.01692 & $0.173 \%$ \\
\hline
\end{tabular}

\section{Gravity/Gravitation Models in Combination with the Ellipsoidal World}

To assess the constant gravity and constant gravitation models on the ellipsoid, one must decide whether the constant acts along the geocentric unit vector or the normal to the surface (geodetic). Except at the equator and the pole, the geocentric unit vector is not normal to the surface of an ellipsoid. Gravity is defined as a surface normal acceleration, and the gravity constant is applied along the geodetic unit vector. Gravitation, on the other hand, is normally expected to act along the geocentric unit vector. However, the equatorial bulge causes the gravitation vector to deviate from the geocentric unit vector. To determine whether the geocentric or geodetic direction is the best option, the mean error of both options were computed using the mean gravitation as the global constant. The geocentric model produces a mean error that is $6.9 \times 10^{-6} \mathrm{~m} / \mathrm{s}^{2}$ larger than the geodetic model. The mean gravitation and mean gravity of the geocentric model also differ from the mean gravity and gravitation of the equipotential ellipsoid by $3 \times 10^{-5} \mathrm{~m} / \mathrm{s}^{2}$ each; the geodetic model matches both mean gravity and gravitation of the equipotential ellipsoid. Manipulating the global gravitation constant in the geocentric model to match the mean gravity of the equipotential ellipsoid worsens the mean error. Based on these results, gravitation constants should be applied to the geodetic 
unit vector. These results were foreshadowed by the unexpectedly low errors of constant gravity with the sphere, whose gravitation is normal to the surface (see section IV.B).

Pairing a non-rotating ellipsoid model with a constant gravity model has the same results as the non-rotating sphere and flat-world. The results of the rotating ellipsoid with a constant gravitation model can still differ from the rotating sphere because the centrifugal acceleration will have different normal and tangential components on the surface of an ellipse and the surface distance to the Earth's z-axis is different. However, the small eccentricity of the Earth limits the maximum difference in the centrifugal acceleration to $4 \times 10^{-5} \mathrm{~m} / \mathrm{s}^{2}$ at the equator. So the errors of the constant gravitation model on the sphere and ellipsoid are equivalent. Because the constants are applied to the surface normal on both the ellipse and sphere, the error growth of the constant gravity and gravitation models with height is equivalent on both the ellipsoid and the sphere near the surface; and the height limits for maintaining the minimum fidelity are the same.

Pairing the point-mass gravitation model with the rotating ellipsoid does produce different errors than point-mass gravitation with the rotating sphere. One does apply point-mass gravitation to the geocentric unit vector because the center of the ellipsoid is the world's center of mass. The resulting gravitation vector will have both a normal and a tangential component on the surface; this differs from the sphere where gravitation has only a normal component. The deviation of the gravitation vector from the normal is the difference between the geodetic $(\phi)$ and geocentric $\left(\phi_{\mathrm{g}}\right)$ latitudes. The resulting surface gravitation components are:

$$
\gamma_{n}^{s}=-\frac{G M}{a^{2}}\left[\frac{\left(1-e^{2} \sin ^{2} \phi\right)^{2}}{\left(1+e^{2}\left(e^{2}-2\right) \sin ^{2} \phi\right)^{3 / 2}}\right] \quad \gamma_{t}^{s}=\frac{G M}{a^{2}}\left[\frac{e^{2} \sin \phi \cos \phi}{\left(1+e^{2}\left(e^{2}-2\right) \sin ^{2} \phi\right)^{3 / 2}}\right]
$$

where $\gamma_{\mathrm{n}}^{\mathrm{s}}$ is the surface normal gravitation and $\gamma_{\mathrm{t}}^{\mathrm{s}}$ is the surface tangential gravitation. For the Earth, the mean of $\gamma_{\mathrm{n}}^{\mathrm{s}}$ is $-9.82023 \mathrm{~m} / \mathrm{s}^{2}$ and matches the mean gravitation of equipotential ellipsoid (error $<1 \times 10^{-9}$ ). The mean of $\gamma_{\mathrm{t}}^{\mathrm{s}}$ is $0.02200 \mathrm{~m} / \mathrm{s}^{2}$ and is nearly double the mean surface tangential component of centrifugal acceleration $(-0.01133$ $\mathrm{m} / \mathrm{s}^{2}$ ). The result was predicted by the sphere combined with the geopotential gravitation model (see section IV.B). The sphere with the geopotential model produces a $\mathrm{g}_{\mathrm{t}}^{\mathrm{s}}$ of $-0.02194 \mathrm{~m} / \mathrm{s}^{2}$ (toward the equator). The sphere with geopotential model represents the absence of gravitation modeling errors in the presence of surface modeling errors. A high fidelity surface model must, therefore, deflect radial gravitation slightly toward the pole from the normal to produce a counteracting tangential acceleration. The ellipsoid with point-mass gravitation represents the absence of surface modeling errors in the presence of gravitation modeling errors and demonstrates that the ellipsoid surface does deflect the radial gravitation to produce a counteracting tangential acceleration. The resulting mean tangential gravity at the surface $\left(\mathrm{g}_{\mathrm{t}}^{\mathrm{s}}\right)$ is $0.01073 \mathrm{~m} / \mathrm{s}^{2}$ toward the pole, since gravitation modeling errors remain. The rotating ellipsoid with point-mass gravitation exhibits a $\mathrm{g}_{\mathrm{t}}^{\mathrm{s}}$ of similar magnitude as the sphere but in the opposite direction.

The ellipsoid with point-mass gravitation produces a mean normal surface gravity of $-9.79764 \mathrm{~m} / \mathrm{s}^{2}$ which matches the theoretical mean surface gravity. Predicted gravity ranges from $-9.76437 \mathrm{~m} / \mathrm{s}^{2}$ at the equator to $-9.86432 \mathrm{~m} / \mathrm{s}^{2}$ at the pole. Table 5 displays the surface gravity errors. The errors remain within the minimum fidelity. However, the surface errors are worse than the constant gravitation model. The point-mass gravitation model does not produce lower errors than constant gravitation below $4 \mathrm{~km}(\sim 13,000 \mathrm{ft})$ and should not be used for simulations that will remain below this height. For a given height above the surface, the point-mass gravitation also performs worse on the ellipsoid (mean error $\sim 0.012 \mathrm{~m} / \mathrm{s}^{2}$ ) than the sphere (mean error $\sim 0.0046 \mathrm{~m} / \mathrm{s}^{2}$ ). However, only simulations, which can ignore the inertial position errors of the sphere, can take advantage of the sphere's improved gravity modeling with point-mass gravitation.

Table 5 Surface Gravity Errors of Point-Mass Gravitation with the Rotating Ellipsoid

\begin{tabular}{|l|r|r|}
\cline { 2 - 3 } \multicolumn{1}{c|}{} & Absolute Error $\left(\mathrm{m} / \mathrm{s}^{2}\right)$ & Percentage Error \\
\hline Equatorial Gravity & -0.01596 & $-0.163 \%$ \\
\hline Polar Gravity & 0.03214 & $0.328 \%$ \\
\hline Mean Error & 0.01231 & $0.126 \%$ \\
\hline
\end{tabular}

The ellipsoid with the geopotential gravitation model represents the ideal surface gravity model that has been used to compare other combinations. Therefore, we should expect no errors in examining the combination. However, in practice, one may encounter very small errors when using geopotential models that were not the basis for defining the ellipsoid. Obtaining the theoretical surface gravity of a world does not necessarily require a complex implementation of a geopotential model. As discussed in section III.B.4, a truncated series using only the even zonal harmonics can approximate the theoretical gravitation field around the ellipsoid. Table 6 shows the predicted gravity values using the first four even zonal harmonics. The mean surface gravity, the equatorial gravity, and the polar gravity match the published values from WGS84. The surface tangent components of the radial and longitudinal gravitation sum to $0.01133 \mathrm{~m} / \mathrm{s}^{2}$ and cancel out the mean surface tangent component of centrifugal acceleration. 
The magnitude $\mathrm{g}_{\mathrm{t}}^{\mathrm{s}}$ remains below $1 \times 10^{-12} \mathrm{~m} / \mathrm{s}^{2}$ over the surface. The geopotential model produces essentially no tangential acceleration due to gravity on the surface of the rotating ellipsoid. Unlike the point-mass gravitation model, the truncated geopotential model does not exhibit worse performance than lower fidelity models under some circumstances. The rotating ellipsoid with the geopotential gravitation model is the only combination that does not produce a net tangential component of gravity on the surface and is also not limited to near-surface simulations. The truncated geopotential model greatly improves gravity modeling on the ellipsoid for a modest increase in computation. The increased fidelity benefits orbiting simulations since it models the effects of the equatorial bulge. On the ellipsoid, simulations should use the truncated geopotential model in preference to the point-mass gravitation model as a low fidelity option to the full geopotential model.

Table 6 Surface Gravity Statistics for Truncated Geopotential Model on a Rotating Ellipsoid

\begin{tabular}{|lr|r|}
\cline { 2 - 3 } \multicolumn{1}{c|}{} & Predicted Value $\left(\mathrm{m} / \mathrm{s}^{2}\right)$ & $\begin{array}{c}\text { Error to Published } \\
\text { WGS84 Value }\left(\mathrm{m} / \mathrm{s}^{2}\right)\end{array}$ \\
\hline Equatorial Normal Gravity & -9.7803253359 & 0.0 \\
\hline Polar Normal Gravity & -9.8321849379 & $-1 \times 10^{-9}$ \\
\hline Mean Normal Gravity on Surface & -9.7976432225 & $-3 \times 10^{-9}$ \\
\hline Mean Tangential Gravity on Surface & $3.8 \times 10^{-14}$ & \\
\hline Mean Normal Gravitation on Surface & -9.8202283499 & \\
\hline $\begin{array}{l}\text { Mean Tangential Acceleration on } \\
\text { Surface from Radial Gravitation }\end{array}$ & 0.0219946903 & \\
\hline $\begin{array}{l}\text { Mean Tangential Acceleration on } \\
\text { Surface from Latitudinal Gravitation }\end{array}$ & -0.0106641372 & \\
\hline Mean Error of Normal Gravity & & \\
\hline
\end{tabular}

\section{Conclusion}

Aerospace simulations that interact with the surface of the world perform ascent, landing, atmospheric flight, or surface travel. Simulation studies of these vehicles may begin with low fidelity models in the concept stage and progress to higher fidelity models during the engineering lifecycle. Increasing the fidelity of the world model and the gravity or gravitation model independently does not necessarily lead to an overall increase in fidelity for near surface operations. The combination may produce errors that overshadow the increased fidelity of each individual model. Various combinations of world models and gravity/gravitation models were compared against the "minimum fidelity" which represents the maximum and mean errors that occur when no higher fidelity effects are modeled. From this comparison, an order of increasing fidelity for surface-interacting simulations emerges:

1. Flat world with a global gravity constant (altitude limited to $1.9 \mathrm{~km}$ )

2. Flat world with origin-based gravity constant (altitude limited to $7.4 \mathrm{~km}$ )

3. Flat world with free-air reduction (origin-based gravity constant preferred)

4. Non-rotating sphere or ellipsoid with a global gravity constant (altitude limited to $1.9 \mathrm{~km}$ )

5. Non-rotating sphere or ellipsoid with free air reduction (global gravity constant)

6. Rotating sphere or ellipsoid with a global gravitation constant (altitude limited to $7.4 \mathrm{~km}$ )

7. Rotating sphere with point-mass gravitation

8. Rotating ellipsoid with geopotential model reduced to even zonal harmonics and truncated

9. Rotating ellipsoid with a complete geopotential model

The list assumes that world modeling fidelity has greater weight than gravity modeling fidelity. Combinations not on the list should be avoided because they exhibit worse fidelity than simpler combinations under some or all circumstances. It is perhaps not a surprise that the investigation found better performance in combinations where the underlying physical assumptions of the gravitation model match the world model, i.e. flat world with free-air reduction, rotating sphere with point-mass gravitation, and rotating ellipsoid with geopotential gravitation model.

Some lessons also emerged from the investigation. First, it is important to understand that gravity and gravitation differ. Gravity is a combination of gravitation and the centrifugal acceleration due to the world's rotation. Non-rotating worlds should be combined with gravity models, and rotating worlds should be combined with gravitation models. The other combinations either eliminate or exaggerate the centrifugal acceleration. Next, standard gravity is defined as a unit of measure, not a modeling parameter for Earth's gravity. However, it performs well as a global constant for the constant gravity model and bests mean gravity as a global constant by producing a range of errors within the minimum fidelity. For all world models, both the gravity and gravitation constants should be applied to the geodetic unit vector; using the geodetic unit vector generates lower surface gravity errors. For altitudes below $15.9 \mathrm{~km}$, the free-air reduction for gravity is a linear model for gravity that performs as well or better than 
"point-mass" formulations of gravity with less computation. From the perspective of gravity modeling, the equatorial radius and the average of the equatorial and polar radii are poor choices for the Earth's radius in the sphere model. The mean radius, radius of equal surface area (to the ellipsoid), and radius of equal volume all generate a mean gravity within $1 \times 10^{-5} \mathrm{~m} / \mathrm{s}^{2}$ of the theoretical mean and are preferred choices. Rotating worlds produce a surface tangent component of centrifugal acceleration; only the rotating ellipsoid with geopotential gravitation model produces a counteracting tangential component of gravitation that leaves a net zero tangential component of gravity.

Though the formulas and methods used in this paper can be applied to any world, the analysis was performed on the Earth whose gravity field is well understood. It is possible that the analysis of another world might produce some different conclusions.

\section{References}

1 “Department of Defense World Geodetic System 1984”, National Imagery and Mapping Agency, NIMA TR8350.2, Third Edition, Amendment 1, 3 January 2000.

2 Moritz, H. “Geodetic Reference System 1980”, Journal of Geodesy, Vol. 74, No.1, 2000, pp. 128-133.

3 Hofmann-Wellenhof, Bernhard and Moritz, Helmut, Physical Geodesy, SpringerWeinNewYork, New York, 2005, ISBN 3-211-23584-1. Sections Preface, 1.7, 2.1, 2.10.

4 "Comptes Rendus de la 3e CGPM (1901)", 3e Conférence Générale des Poids et Mesures, Paris, 1901. Also available at URL: http://www.bipm.fr/en/CGPM/db/3/2/ [Cited Jul 19, 2006]

5 Halliday, D. and Resnick, R. Fundamentals of Physics, John Wiley and Sons, New York, 1981, inside cover.

${ }^{6}$ Eide, Arvid R., Jenison, Roland D., Mashaw, Lane H., and Northup, Larry L. Engineering Fundamentals and Problem Solving, McGraw-Hill, New York, 1986, Second Edition, ISBN 0-07-019318-5, Appendix D.

7 "StarChild Question of the Month for February 2001", National Aeronautics and Space Administration, URL: http://starchild.gsfc.nasa.gov/docs/StarChild/questions/question30.html, [cited July 5, 2006].

8 Soukhanov, Anne H. (Ed.) Webster's II New Riverside University Dictionary, The Riverside Publishing Company, Boston, 1994, ISBN 0-395-33957-X.

9 WordNet [online database] Princeton University Cognitive Science Laboratory, Princeton, URL: http://wordnet.princeton.edu/ [cited July 19, 2006].

10 "Gravity", Encyclopedia Britannica Online [online database], Encyclopedia Britannica, Chicago, URL: http://www.britannica.com/eb/article-9037796 [cited July 19, 2006]

${ }^{11}$ Allen, William H. (Ed.), "Dictionary of Technical Terms for Aerospace Use", National Aeronautics and Space Administration, Washington D.C., NASA SP-7, First Edition, 1965. Also available as an online database at URL: http://roland.lerc.nasa.gov/ dglover/dictionary/content.html [cited July 19, 2006].

${ }^{12}$ Li, Xiong and Götze Hans-Jurgën, "Tutorial: Ellipsoid, geoid, gravity, geodesy, and geophysics”, Geophysics, Vol. 66, No. 6, November-December 2001, pp. 1660-1668.

13 "Space Environment", European Cooperation for Space Standardization, ECSS-E-10-04A, 21 January, 2000.

${ }^{14}$ Lemoine (et. al.), "The Development of the Joint NASA GSFC and the National Imagery and Mapping Agency (NIMA) Earth Geopotential Model EGM96", National Aeronautics and Space Administration, Washington D.C., NASA-TP-1998206861, July, 1998.

15 Lundberg, John B. and Schutz, Bob E. "Recursion Formulas of Legendre Functions for Use with Nonsingular Geopotential Models", Journal of Guidance, Vol. 11, No. 1, January-February 1988, pp. 31-38.

${ }^{16}$ Pines, Samuel, "Uniform Representation of the Gravitational Potential and its Derivatives", AIAA Journal, Vol. 11, No. 11, 1973, pp. 1508-1511.

${ }^{17}$ Bate, Roger R., Mueller, Donald D., and White, Jerry E. Fundamentals of Astrodynamics, Dover Publications, New York, 1971, ISBN 0-486-60061-0, Section 9.7. 